\section{ESTABLISHING A SATELLITE SIMULATION SUITE ON THE NEONATAL INTENSIVE CARE UNIT}

Gabrielle Simpson, Kathryn Martinello, Amy Gilbert. Great Ormond Street Hospital

\subsection{6/archdischild-2020-gosh.92}

Introduction In situ simulation has long been considered the most favourable method of delivering immersive simulation. It allows candidates to rehearse clinical scenarios in a familiar environment and takes into consideration systems testing which can highlight potential latent safety threats. In situ simulation can further develop candidates' technical skills and contribute to team building and communication skills.

In a high intensity environment such as neonatal intensive care (NICU), being prepared for rapid deterioration in acutely unwell patients is a necessity. Great Ormond Street Hospital NICU is a level 3 teaching unit with a high turnover of medical and nursing staff, and thus building key team working relationships can be challenging.

Methods A manikin from the Clinical Simulation Centre (CSC) was loaned to the NICU and a satellite simulation suite was established in a NICU cubicle. The manikin is stored in a portable cabinet to allow ease of movement throughout the unit. The suite was stocked with expired consumables to minimise waste and provide the necessary equipment needed for clinical scenarios.

We developed a curriculum and regular weekly schedule factored into the unit's daily demands. This allows the clinical staff to pre-allocate candidates and ensures equal opportunities for clinical staff to attend.

Results We faced some challenges initially including identifying the ideal time and ensuring staff engagement. After overcoming these challenges, the programme has become well established. Staff are better engaged with the programme and timings are integrated into the unit's culture. Scenarios have been written to address patient safety incidents and identified knowledge gaps.

Discussion Despite facing initial challenges of staff engagement and incorporating the programme into the unit's culture, the satellite suite has been a huge success and has impacted on ward culture and patient safety.

\section{HANDOVER RELATED ANXIETY}

Sevasti Konstantinidou, Nikita Patel, Jayaram Sivaraj , Manal Dhaiban, Dylan Wills, Stavros Loukogeorgakis, Ceri Jones. Great Ormond Street Hospital

10.1136/archdischild-2020-gosh.93

Background Handover is a vital clinical skill to transfer information to ensure continuity of care for patients. However, handover can be stressful with significant effect on clinicians. It is important to acknowledge participants well-being and level of expectation. This project aims to assess and address handover as cause of anxiety for the clinical team.

Methods We have conducted a quality improvement project assessing the impact of handover to participants well-being, utilising a verified questionnaire. Following the results, changes were implemented to provide a standardised structure to handover, providing a framework and expectation for the process with an aim to improve clinicians' satisfaction and quality of handover.

Results Twenty questionnaires were initially completed regarding handover. Most of the participants felt that handover was a source of anxiety. Seventy-five percent of the consultant respondents felt anxiety related to time pressure and the need to attend to clinical and nonclinical responsibilities. The majority $(66 \%)$ of the registrar respondents had anxiety related to leading and receiving handover. The junior team appeared to be the group less effected by handover. Following recognition of this and implementation of improvements, reported anxiety was reduced to $20 \%$ among all team members.

Conclusions We have identified the handover as a stressful period of the working day. The reason for anxiety is very interesting as it varies for different members of the team. Initial interventions had positive effect on participants. We aim to continue assessing, teaching 'handover communication skills' and refining handover process to improve mental health linked to handover.

\section{GERMLINE MOSAICISM - WHEN A RARE DISEASE RECURS}

${ }^{1}$ Tanya Lam, ${ }^{2}$ Shamima Rahman, ${ }^{3}$ Ajith Kumar. ${ }^{1}$ Clinical Fellow, Clinical Genetics, GOSH: ${ }^{2}$ Professor and Honorary Consultant in Mitochondrial disorders, GOSH; ${ }^{3}$ Consultant, Clinical Genetics, GOSH

\subsection{6/archdischild-2020-gosh.94}

Rare autosomal dominant and $\mathrm{X}$ linked genetic disorders are often caused by new mutations. Both parents are healthy in these instances and they are counselled that the risk of recurrence in their future children is likely to be low (usually less than 1\%). However, in some of these families, one parent may have a mutation confined to some cells including testicular or ovarian cells (germline or gonadal mosaicism). Whilst rare, germline mosaicism may significantly change the risk of recurrence in future offspring, with implications for genetic counselling. We review families who have multiple affected children due to germline mosaicism, discuss conditions in which germline mosaicism is more common and a current study that aims to stratify risk using NGS (Next Generation Sequencing) on multiple cell lines in both parents.

\section{ASPIRANT NURSES - THE JOURNEY OF WELCOMING A NOVEL ROLE INTO THE NURSING FAMILY AT GREAT ORMOND STREET HOSPITAL (GOSH)}

Kate Prosser. Great Ormond Street Hospital

\subsection{6/archdischild-2020-gosh.95}

Aim As part of the national response to Covid-19, emergency legislation was passed allowing final year nursing students to undertake paid placements to support the NHS. This paper describes the approach taken by the Nursing Workforce Team to facilitate the successful introduction of this novel, nationally recognised role to GOSH.

Approach Collaboration with internal and external stakeholders including North Central London Capital Nurse Returner Bureau, GOSH Nursing and Non-Medical Education Team (NNMET), Higher Education Institutions (HEIs), Health Education England (HEE), and GOSH Human Resources (HR) in order to divide and agree on actions to enable us to host as many Aspirant Nurses as possible. 